\title{
Convolutional neural networks for the design and analysis of non-fullerene acceptors
}

\author{
Shi-Ping Peng and Yi Zhao* \\ State Key Laboratory for Physical Chemistry of Solid Surfaces, Collaborative Innovation \\ Center of Chemistry for Energy Materials, Fujian Provincial Key Lab of Theoretical and \\ Computational Chemistry, and College of Chemistry and Chemical Engineering, Xiamen \\ University, Xiamen 361005, China \\ E-mail: yizhao@xmu.edu.cn
}

\section{Modeling}

The molecular generation and prediction models are built with Pytorch (v1.1) and run on NVIDIA 1080Ti. Both of our models are trained with the batch size of 32 and the size of each embedding vector is 32 . Adam is taken as an optimizer with the initializing learning rate of 0.001 . For convolutional layers, the kernel size is taken as 3 , the stride is 1 and the dilation factor is $2^{n-1}$ for the n-th layer. In 200 epochs, the learned parameters of the models, with the best performance in validation set, are saved. The training of generative model (include sampling 100000 molecules) takes about 25 thousand seconds of CPU time. The training of prediction model takes about 12 thousand seconds of CPU time. All of the codes are upload at https://github.com/PSPhi/CNN-for-NFA. 


\section{The comparian of generative model}

The MOlecular SEtS (MOSES), ${ }^{1}$ a benchmarking platform to evaluate the diversity and quality of generated molecules with a set of metrics, is used for the comparison of molecular generation models with a dataset refined from the ZINC database. Table S1 lists the metrics for the generative models based on character-level recurrent neural network (CharRNN), variational autoencoder (VAE), adversarial autoencoder (AAE), junction tree variational autoencoder (JTN-VAE) and convolutional neural network (CNN). The comparison table and the source code of the platform were downloaded at December, 2018 from https:// github.com/molecularsets/moses.

Besides standard uniqueness and validity metrics, MOSES provides other metrics to access the overall quality of generated molecules. Fragment similarity (Frag) and Scaffold similarity (Scaff) are cosine distances between vectors of fragment or scaffold frequencies correspondingly of the generated and test sets. Nearest neighbor similarity (SNN) is the average similarity of generated molecules to the nearest molecule from the test set. Internal diversity (IntDiv) is an average pairwise similarity of generated molecules. Fréchet ChemNet Distance (FCD) measures the difference in distributions of last layer activations of ChemNet.

Table S1: Metrics for molecular generation models

\begin{tabular}{|c|c|c|c|c|c|c|}
\hline \multicolumn{2}{|c|}{ Model } & CharRNN & VAE & AAE & JTN-VAE & CNN \\
\hline \multicolumn{2}{|c|}{ Valid $(\uparrow)$} & $\mathbf{0 . 9 5 9 8}$ & 0.9528 & 0.9341 & 1 & 0.9419 \\
\hline \multicolumn{2}{|c|}{ Unique@1k( $\uparrow)$} & $\mathbf{1}$ & $\mathbf{1}$ & $\mathbf{1}$ & 0.998 & 0.998 \\
\hline \multicolumn{2}{|c|}{ Unique@10k( $(\uparrow)$} & $\mathbf{0 . 9 9 9 3}$ & 0.9992 & 1 & 0.9972 & 0.9851 \\
\hline \multirow{2}{*}{ FCD $(\downarrow)$} & Test & 0.3233 & 0.2540 & 1.3511 & 4.3769 & $\mathbf{0 . 2 5 0 4}$ \\
\cline { 2 - 7 } & TestSF & 0.8355 & 0.6959 & 1.8587 & 4.6299 & $\mathbf{0 . 6 6 0 2}$ \\
\hline \multirow{2}{*}{ SNN $(\downarrow))$} & Test & 0.4606 & 0.4684 & 0.4191 & $\mathbf{0 . 3 9 0 9}$ & 0.4791 \\
\cline { 2 - 7 } & TestSF & 0.4492 & 0.4547 & 0.4113 & $\mathbf{0 . 3 9 0 2}$ & 0.4617 \\
\hline \multirow{2}{*}{ Frag $(\uparrow)$} & Test & 0.9977 & 0.9978 & 0.9865 & 0.9679 & $\mathbf{0 . 9 9 8 7}$ \\
\cline { 2 - 7 } & TestSF & 0.9962 & 0.9963 & 0.9852 & 0.9699 & $\mathbf{0 . 9 9 6 9}$ \\
\hline \multirow{2}{*}{ Scaff $(\uparrow)$} & Test & 0.7964 & $\mathbf{0 . 8 2 7 7}$ & 0.6637 & 0.3868 & 0.8166 \\
\cline { 2 - 7 } & TestSF & 0.1281 & 0.0925 & $\mathbf{0 . 1 5 3 8}$ & 0.1163 & 0.0454 \\
\hline \multicolumn{2}{|c|}{ IntDiv $(\uparrow)$} & $\mathbf{0 . 8 5 6 1}$ & 0.8548 & 0.8531 & 0.8495 & 0.8558 \\
\hline \multicolumn{2}{|c|}{ Filters $(\uparrow)$} & 0.9920 & 0.9925 & 0.9759 & 0.9566 & $\mathbf{0 . 9 9 5 8}$ \\
\hline
\end{tabular}




\section{The learning curve of prediction model}

The mean absolute errors of HOMO and LUMO energies for validation set are $0.051 \mathrm{eV}$, $0.053 \mathrm{eV}$ and the error of PCE is about $0.30 \%$. The mean absolute errors of HOMO and LUMO energies for test set are $0.053 \mathrm{eV}, 0.055 \mathrm{eV}$ and the error of PCE is about $0.31 \%$. The learning curves are shown in Figure S1.
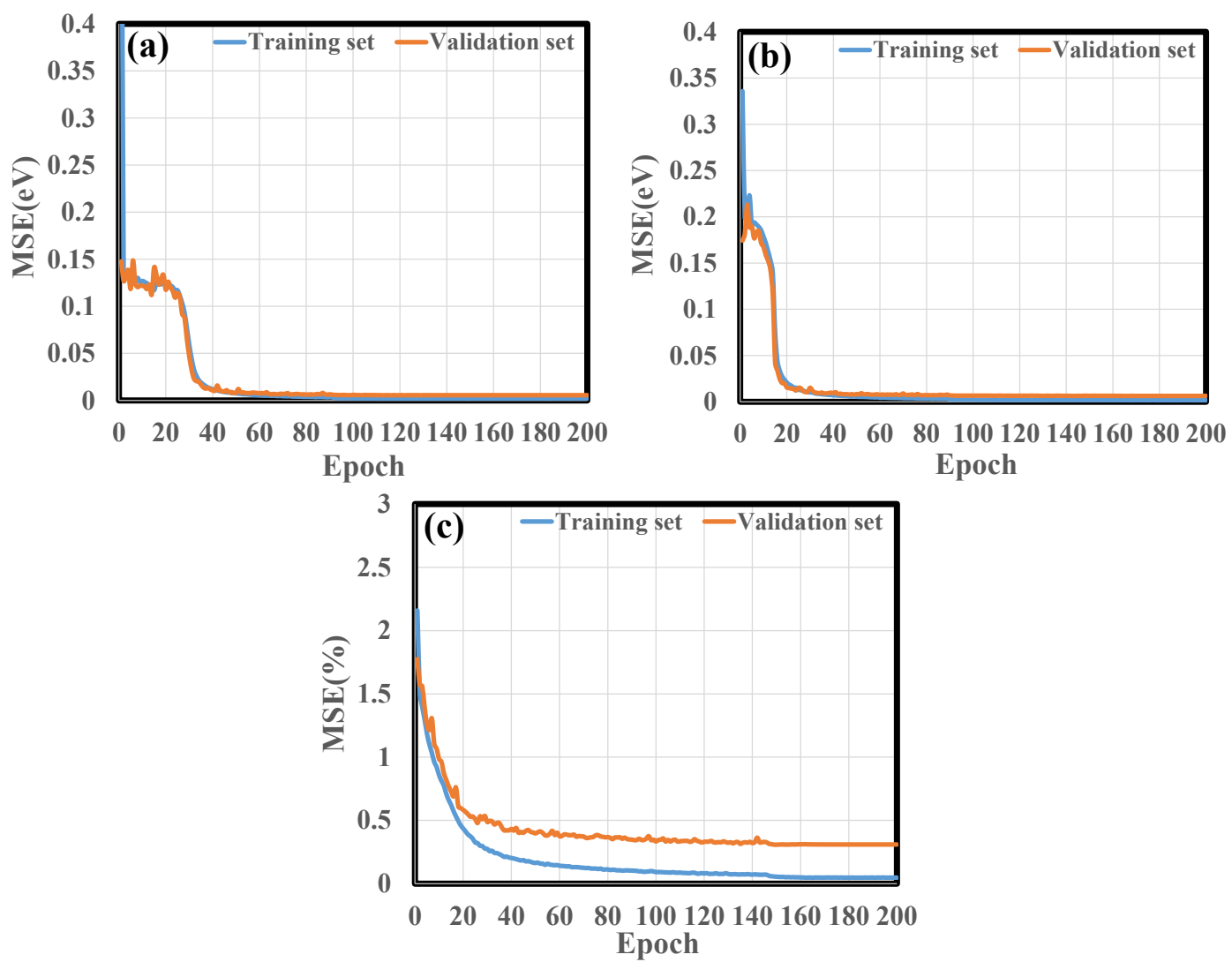

Figure S1: The learning curves of (a)HOMO, (b)LUMO and (c)PCE.

\section{The orbital composition analysis}

The contribution of each fragment to the HOMO/LUMO is calculated by natural atomic orbital method with Multiwfn 3.6. The contribution of the atomic orbital is calculated by multiplying the corresponding matrix elements of the molecular orbital coefficient matrix based on NAO by $100 \%$. Multiwfn does not generate this matrix itself. The user needs to 
use the output file of the NBO program (such as Gaussian) containing this matrix as the input file of Multiwfn. For Gaussian, write the route section to pop=nboread and write a line to the end of the molecular coordinates with \$NBO NAOMO \$END in input file. After calculation, the Gaussian output file can be used as an input file for NAO analysis of Multiwfn.

\section{References}

(1) Polykovskiy, D.; Zhebrak, A.; Sanchez-Lengeling, B.; Golovanov, S.; Tatanov, O.; Belyaev, S.; Kurbanov, R.; Artamonov, A.; Aladinskiy, V.; Veselov, M.; Kadurin, A.; Nikolenko, S.; Aspuru-Guzik, A.; Zhavoronkov, A. Molecular Sets (MOSES): A Benchmarking Platform for Molecular Generation Models. http://arxiv.org/abs/1811.12823, 2018. 